\title{
The Combined Reproducing Kernel Method and Taylor Series for Solving Weakly Singular Fredholm Integral Equations
}

\author{
Azizallah Alvandi ${ }^{1}$, Mahmoud Paripour ${ }^{2}$ \\ ${ }^{1}$ Department of Mathematics, Hamedan Branch, Islamic Azad University, Iran \\ ${ }^{2}$ Department of Mathematics, Hamedan University of Technology, Iran
}

\begin{tabular}{l}
\hline Article Info \\
\hline Article history: \\
Received Mei 22, 2016 \\
Revised Aug 10, 2016 \\
Accepted Aug 22, 2016 \\
\hline
\end{tabular}

\begin{abstract}
In this paper, a numerical method is proposed for solving weakly singular Fredholm integral equations in Hilbert reproducing kernel space (RKHS). The Taylor series is used to remove singularity and reproducing kernel function are used as a basis. The effectiveness and stability of the numerical scheme is illustrated through two numerical examples.
\end{abstract}

\section{Keyword:}

Fredholm integral

Reproducing kernel

Taylor series

Weakly singular

\section{Corresponding Author:}

Azizallah Alvandi,

Department of Mathematics,

Hamedan Branch,

Islamic Azad University, Hamedan, Iran.

Email: Alvandya@gmail.com

\section{INTRODUCTION}

Weakly singular Fredholm integral equations (WSFIEs) have many applications in problems of applied sciences, mathematical physics, astrophysics and solid mechanics. The numerical solvability of these equations and other related equations have been pursued by several authors and solved by many numerical methods such as generalization of the Euler-Maclaurin summation formula [1], application of homotopy perturbation method [2], differential transform method [3], discrete Galerkin method [4], modified HPM method [5], SCW method [6], spectral methods [7], fractional linear multi-step methods [8], Jacobi spectral method [9] and other methods occured in [10-14].

Recently, based on reproducing kernel theory, the reproducing kernel method (RKM) has been successfully applied to integral equations, Hilbert type singular integral equations of the second kind [15], Fredholm integral equation of the first kind [16], singular integral equation with cosecant kernel [17], the reproducing kernel method has been presented and developed in [18-25].

In this letter, a numerical scheme by using reproducing kernel space and Taylor series to solve the following weakly singular Fredholm integral equation is provided:

$$
u(x)=f(x)+\int_{0}^{1} k(x, t) u(t) \mathrm{d} t, \quad 0 \leq x \leq 1,
$$

where kernel $k(x, t)=\frac{1}{(1-t)^{\alpha}}$ with the assumption $0<\alpha<1$, is weakly singular and $u(x)$ is the unknown function to be determined. 
This paper is organized as five sections including the introduction. In the next section, the reproducing kernel spaces are presented in order to construct reproducing kernel functions in the space $W_{2}^{m}[0,1]$. Equation (1) is converted into an equivalent equation and the representation of approximate solution for Fredholm integral equations with a weakly singular kernel is obtained in Section 3. The numerical examples are presented to demonstrate the accuracy of the method in Section 4 . The last section is a brief conclusion.

\section{A REPRODUCING KERNEL HILBERT SPACE $W_{2}^{m}[0,1]$}

The function space $W_{2}^{m}[0,1]$ is defined as follows:

Definition 2.1. $W_{2}^{m}[0,1]=\left\{u(x) \mid u^{(m-1)}(x)\right.$ is an absolutely continuous real value function, $\left.u^{(m)}(x) \in L^{2}[0,1]\right\}$. The inner product and norm in $W_{2}^{m}[0,1]$ are defined respectively by

$$
\langle u, v\rangle_{W_{2}^{m}}=\sum_{i=0}^{m-1} u^{(i)}(0) v^{(i)}(0)+\int_{0}^{1} u^{(m)}(x) v^{(m)}(x) \mathrm{d} x
$$

And

$$
\|u\|_{m}={\sqrt{\langle u, u\rangle_{m}}}, \quad u, v \in W_{2}^{m}[0,1]
$$

In general, the function space $W_{2}^{m}[0,1]$ is a reproducing kernel space and its reproducing kernel $R_{x}(y)$ has the following reproducing property

$$
\left\langle u(y), R_{x}(y)\right\rangle=u(x), \quad \forall u \in W_{2}^{m}[0,1]
$$

The reproducing kernel $R_{x}(y)$ can be denoted by

$$
R_{x}(y)= \begin{cases}\sum_{i=1}^{2 m} c_{i}(y) x^{i-1}, & x \leq y, \\ \sum_{i=1}^{2 m} d_{i}(y) x^{i-1}, & x>y,\end{cases}
$$

where coefficients $c_{i}(y), d_{i}(y),\{i=1,2, \cdots, 2 m\}$, could be obtained by solving the following equations

$$
\begin{aligned}
& \left.\frac{\partial^{i} R_{y}(x)}{\partial x^{i}}\right|_{x=y^{+}}=\left.\frac{\partial^{i} R_{y}(x)}{\partial x^{i}}\right|_{x=y^{-}}, \quad i=0,1,2, \cdots, 2 m-2, \\
& (-1)^{m}\left(\left.\frac{\partial^{2 m-1} R_{y}(x)}{\partial x^{2 m-1}}\right|_{x=y^{+}}-\left.\frac{\partial^{2 m-1} R_{y}(x)}{\partial x^{2 m-1}}\right|_{x=y^{-}}\right)=1,
\end{aligned}
$$




$$
\left\{\begin{array}{c}
\frac{\partial^{i} R_{y}(0)}{\partial x^{i}}-(-1)^{m-i-1} \frac{\partial^{2 m-i-1} R_{y}(0)}{\partial x^{2 m-i-1}}=0, \quad i=0,1, \cdots, m-1, \\
\frac{\partial^{2 m-i-1} R_{y}(1)}{\partial x^{2 m-i-1}}=0, \quad i=0,1, \cdots, m-1 .
\end{array}\right.
$$

\section{SOLVING EQUATION (1) IN THE REPRODUCING KERNEL SPACE}

3.1. An Equivalent Transformation of Equation (1)

In this section, for solving Equation (1) an equivalent transformation of Equation (1) is proposed. Consider the integral equation with the given conditions in relation (1). With the Taylor series expansion of $u(t)$ based on expanding about the given point $x$ belonging to the interval 0,1 , we have the Taylor series approximation of $u(t)$ in the following form

$$
u(t)=u(x)+(t-x) u^{\prime}(x)+\frac{(t-x)^{2}}{2} u^{\prime \prime}(x)+\cdots+\frac{(t-x)^{n}}{n !} u^{(n)}(x)+\frac{(t-x)^{n+1}}{(n+1) !} u^{(n+1)}\left(\zeta_{x, t}\right),
$$

where $\zeta_{x, t}$ is between $\mathrm{x}$ and t. By substituting relation (8) into Equation (1), we have

$$
\begin{aligned}
& u(x)-\int_{0}^{1}(1-t)^{-\alpha} \sum_{k=0}^{n} \frac{(t-x)^{k}}{k !} u^{(k)}(x) \mathrm{d} t+E_{n}(x) \\
& =u(x)-\sum_{k=0}^{n} \frac{u^{(k)}(x)}{k !} \int_{0}^{1}(1-t)^{-\alpha}(t-x)^{k} \mathrm{~d} t+E_{n}(x)=f(x),
\end{aligned}
$$

where $u^{(0)}(x)=u(x)$ and $E_{n}(x)=\frac{1}{(n+1) !} \int_{0}^{1}(1-t)^{-\alpha}(t-x)^{n+1} u^{(n+1)}\left(\zeta_{x, t}\right) \mathrm{d} t$. Alternatively, we use the truncated Taylor series of $u(t)$ and solve the following equation

$$
u(x)-\sum_{k=0}^{n} \frac{u^{(k)}(x)}{k !} \int_{0}^{1}(1-t)^{-\alpha}(t-x)^{k} \mathrm{~d} t=f(x),(10)
$$

when $0<\alpha<1, \int_{0}^{1}(1-t)^{-\alpha}(t-x)^{k} \mathrm{~d} t$ is computable for $k=0,1, \cdots, n$. Hence, Equation (10) can be written as following

$$
\sum_{k=0}^{n} a_{k}(x) u^{(k)}(x)=f(x)
$$

\subsection{The Exact and Approximate Solution}

The solution of Equation (11) is given in the reproducing kernel Hilbert space $W_{2}^{m}[0,1](m>n)$, parameter $n$ is related to the number of terms Taylor series that are chosen. We define the operator $\mathrm{L}: W_{2}^{m}[0,1] \rightarrow W_{2}^{m-n}[0,1]$ as

$$
\mathrm{L} u(x)=\sum_{k=0}^{n} a_{k}(x) u^{(k)}(x)
$$

then Equation (11) can be written as 


$$
\mathrm{L}(u)=f(x)
$$

It is clear that $L$ is a bounded linear operator and $L^{*}$ is the adjoint operator of $L$. In order to obtain the representation of the solution of Equation (11), let

$$
\phi_{i}(x)=R_{x_{i}}(x), \quad \psi_{i}(x)=L^{*} \phi_{i}(x)=\left[L_{y} R_{x}(y)\right]\left(x_{i}\right), \quad(i=1,2, \ldots),
$$

where $\left\{x_{i}\right\}_{i=1}^{\infty}$ is dense in the interval $[0,1]$. Hence, one gets

$$
\psi_{i}(x)=\left.\sum_{k=0}^{n} a_{k}\left(x_{i}\right) \frac{\partial^{k} R(x, y)}{\partial y^{k}}\right|_{y=x_{i}}
$$

Theorem 3.2.1. If $\left\{x_{i}\right\}_{i=1}^{\infty}$ is dense in $[0,1]$, then $\left\{\psi_{i}(x)\right\}_{i=1}^{\infty}$ is complete system in $W_{2}^{m}[0,1]$. Proof. If for any $u(x) \in W_{2}^{m}[0,1]$, it has $\left\langle u(x), \psi_{i}(x)\right\rangle=0 \quad i=1,2, \ldots$, namely

$$
\begin{aligned}
& \left\langle u(x), \psi_{i}(x)\right\rangle=\left\langle u(x),\left({\mathrm{L} y R_{x}}_{x}(y)\left(x_{i}\right)\right\rangle\right. \\
& =\mathrm{L}_{y}\left\langle u(x), R_{x}(y)\right\rangle\left(x_{i}\right)=\left[\mathrm{L}_{y} u(y)\right]\left(x_{i}\right)=0 .
\end{aligned}
$$

Note that $\left\{x_{i}\right\}_{i=1}^{\infty}$ is a dense set, hence $\mathrm{L}_{y} u(x) \equiv 0$. It follows that $u(x) \equiv 0$. So the proof of theorem is complete. By Gram-Schmidt process, we obtain an orthogonal basis $\left\{\bar{\psi}_{i}(x)\right\}_{i=1}^{\infty}$ of $W_{2}^{m}[0,1]$, such that

$$
\bar{\psi}_{i}(x)=\sum_{k=1}^{i} \beta_{i k} \psi_{k}(x)
$$

where $\beta_{i k}$ are orthogonal coefficients. In order to obtain $\beta_{i j}$, let

$$
\begin{aligned}
& \psi_{i}(x)=\sum_{k=1}^{i} B_{i k} \bar{\psi}_{k}(x) . \\
& \left\langle\psi_{i}(x), \psi_{i}(x)\right\rangle=\sum_{k=1}^{i-1} B_{i k}^{2}+B_{i i}^{2}, \\
& B_{i i}=\sqrt{\left\langle\psi_{i}(x), \psi_{i}(x)\right\rangle-\sum_{k=1}^{i-1} B_{i k}^{2}} . \\
& \beta_{i i}=\frac{1}{\sqrt{\left\langle\psi_{i}(x), \psi_{i}(x)\right\rangle-\sum_{k=1}^{i-1} B_{i k}^{2}}} . \\
& \beta_{i j}=\beta_{i i}\left(-\sum_{k=j}^{i-1} B_{i k} \beta_{k j}\right) .
\end{aligned}
$$

Theorem 3.2.2 If $u(x)$ is the solution of Equation (1), then

$$
u(x)=\sum_{i=1}^{\infty} \sum_{k=1}^{i} \beta_{i k} f\left(x_{k}\right) \bar{\psi}_{i}(x)
$$

Proof. $u(x)$ can be expanded to Fourier series in term of normal orthogonal basis $\bar{\psi}_{i}(x)$ in $W_{2}^{m}[0,1]$, 


$$
\begin{aligned}
& u(x)=\sum_{i=1}^{\infty}\left\langle u(x), \bar{\psi}_{i}(x)\right\rangle \bar{\psi}_{i}(x)=\sum_{i=1}^{\infty} \sum_{k=1}^{i} \beta_{i k}\left\langle u(x), \psi_{k}(x)\right\rangle \bar{\psi}_{i}(x) \\
& =\sum_{i=1}^{\infty} \sum_{k=1}^{i} \beta_{i k}\left\langle u(x), \mathrm{L}^{*} \varphi_{k}(x)\right\rangle \bar{\psi}_{i}(x)=\sum_{i=1}^{\infty} \sum_{k=1}^{i} \beta_{i k}\left\langle\mathrm{~L} u(x), \varphi_{k}(x)\right\rangle \bar{\psi}_{i}(x) \\
& =\sum_{i=1}^{\infty} \sum_{k=1}^{i} \beta_{i k}\left\langle f(x), \varphi_{k}(x)\right\rangle \bar{\psi}_{i}(x)=\sum_{i=1}^{\infty} \sum_{k=1}^{i} \beta_{i k} f\left(x_{k}\right) \bar{\psi}_{i}(x) .
\end{aligned}
$$

The proof is complete.

By truncating the series of the left-hand side of (19), we obtain the approximate solution of (1)

$$
u_{N}(x)=\sum_{i=1}^{N} \sum_{k=1}^{i} \beta_{i k} f\left(x_{k}\right) \bar{\psi}_{i}(x) .
$$

$u_{N}(x)$ in (21) is the $N$-term intercept of $u(x)$ in (19), so $u_{N}(x) \rightarrow u(x)$ in $W_{2}^{m}[0,1]$ as $N \rightarrow \infty$. Lemma 3.2.1. If $u(x) \in W_{2}^{m}[0,1]$, then there exists a constant $c$ such that $|u(x)| \leq c \mathrm{P} u(x) \mathrm{P}_{m}$.Proof.

$$
|u(x)|=\left|\left\langle u(y), R_{x}(y)\right\rangle\right| \leq\|u(y) \mid\| R_{x}(y) \|_{m},
$$

there exists a constant $c$ such that

$$
|u(x)| \leq c\|u\|_{m}
$$

The proof of the lemma is complete. W

Theorem 3.2.3. Suppose the following conditions are satisfied

(i) $\left\|u_{N}(x)\right\|_{W_{2}^{m}}$ is bounded;

(ii) $\left\{x_{i}\right\}_{i=1}^{\infty}$ is dense in $[0,1]$.

Then $N$-term approximate solution $u_{N}(x)$ converges to the exact solution $u(x)$ of Equation (1) and the exact solution is expressed as

$$
u(x)=\sum_{i=1}^{\infty} B_{i} \bar{\psi}_{i}(x)
$$

where $B_{i}=\sum_{k=1}^{i} \beta_{i k} f\left(x_{k}\right)$.

Proof. (i) The convergence of $u_{N}(x)$ will be proved. From (21), one gets

$$
u_{N}(x)=u_{N-1}(x)+B_{N} \bar{\psi}_{N}(x)
$$

From the orthogonality of $\left\{\bar{\psi}_{i}(x)\right\}_{i=1}^{\infty}$, it follows that

$$
\left\|u_{N}(x)\right\|_{W_{2}^{m}}^{2}=\left\|u_{N-1}(x)\right\|_{W_{2}^{m}}^{2}+\left\|B_{N}\right\|^{2}
$$

The sequence $\left\|u_{N}(x)\right\|_{W_{2}^{m}}$ is monotone increasing. Due to $\left\|u_{N}(x)\right\|_{W_{2}^{m}}$ being bounded, $\left\|u_{N}(x)\right\|_{W_{2}^{m}}$ is convergent as soon as $N \rightarrow \infty$. Then there exists a constant $c$ such that 


$$
\sum_{i=1}^{\infty} B_{i}^{2}=c
$$

let $m>N$, in view of $\left(u_{m}-u_{m-1}\right) \perp\left(u_{m-1}-u_{m-2}\right) \perp \ldots \perp\left(u_{N+1}-u_{N}\right)$, it follows that

$$
\begin{aligned}
& \left\|\left(u_{m}-u_{N}\right)\right\|_{W_{2}^{m}}^{2}=\left\|u_{m}-u_{m-1}+u_{m-1}-u_{m-2}+\ldots+u_{N+1}-u_{N}\right\|_{W_{2}^{m}}^{2} \\
& =\left\|u_{m}-u_{m-1}\right\|_{W_{2}^{m}}^{2}+\left\|u_{m-1}-u_{m-2}\right\|_{W_{2}^{m}}^{2}+\ldots+\left\|u_{N+1}-u_{N}\right\|_{W_{2}^{m}}^{2} \\
& =\sum_{i=N+1}^{m}\left(B_{i}\right)^{2} \rightarrow 0,(N \rightarrow \infty) .
\end{aligned}
$$

Considering the completeness of $W_{2}^{m}[0,1]$, it has

$$
u_{N}(x) \stackrel{\|\|_{W_{2}^{m}}^{m}}{\rightarrow} u(x), \quad N \rightarrow \infty .
$$

(ii) It is proved that $u(x)$ is the solution of Equation (11).

From (22), it follows

$$
\begin{aligned}
(\mathrm{L} u)\left(x_{j}\right) & =\sum_{i=1}^{\infty} B_{i}\left\langle\mathrm{~L} \bar{\psi}_{i}(x), \varphi_{j}(x)\right\rangle \\
& =\sum_{i=1}^{\infty} B_{i}\left\langle\bar{\psi}_{i}(x), \mathrm{L}^{*} \varphi_{j}(x)\right\rangle \\
& =\sum_{i=1}^{\infty} B_{i}\left\langle\bar{\psi}_{i}(x), \psi_{j}(x)\right\rangle,
\end{aligned}
$$

it follows that

$$
\begin{aligned}
\sum_{j=1}^{N} \beta_{N j}(\mathrm{~L} u)\left(x_{j}\right) & =\sum_{i=1}^{\infty} B_{i}\left\langle\bar{\psi}_{i}(x), \sum_{j=1}^{N} \beta_{N_{j}} \psi_{j}(x)\right\rangle_{W_{2}^{m}} \\
& =\sum_{i=1}^{\infty} B_{i}\left\langle\bar{\psi}_{i}(x), \bar{\psi}_{N}(x)\right\rangle_{W_{2}^{m}}=B_{N} .
\end{aligned}
$$

If $N=1$, then $(\mathrm{L} u)\left(x_{1}\right)=f\left(x_{1}\right)$.

If $N=2$ then $\beta_{21}(\mathrm{~L} u)\left(x_{1}\right)+\beta_{22}(\mathrm{~L} u)\left(x_{2}\right)=\beta_{21} f\left(x_{1}\right)+\beta_{22} f\left(x_{2}\right)$.

It is clear that $(\mathrm{L} u)\left(x_{2}\right)=f\left(x_{2}\right)$.

Moreover, it is easy to see by induction that $(\mathrm{L} u)\left(x_{j}\right)=f\left(x_{j}\right)$. Since $\left\{x_{i}\right\}_{i=1}^{\infty}$ is dense on $[0,1]$, for any $x \in[0,1]$

$$
(\llcorner u)(x)=f(x) .
$$

That is, $u(x)$ is the solution of Equation (11) and

$$
u(x)=\sum_{i=1}^{\infty} B_{i} \bar{\psi}_{i}(x) .
$$

The proof is complete. 


\section{NUMERICAL EXAMPLES}

In this section, two examples with exact solutions are given. We take $N=10$, that $N$ is the number of terms of the Fourier series of the unknown function $u(x)$. Parameter $n$ is the number of terms of the Taylor series and we choose $m>n$ for solving these examples.

Example 4.1. We consider the following weakly singular Fredholm integral Equation [1]:

$$
u(x)=x^{2}-\frac{16}{15}+\int_{0}^{1} \frac{u(t)}{\sqrt{1-t}} \mathrm{~d} t, \quad 0 \leq x \leq 1,
$$

with the exact solution $u(x)=x^{2}$.

Let $n=3$ and applying the reproducing kernel method. The comparison between the exact solution and the approximate solution and the absolute errors in spaces $W_{2}^{7}[0,1], W_{2}^{8}[0,1]$ are graphically shown in Figure 1, respectively. The absolute errors between $u(x)$ and $u_{10}(x)$ in spaces $W_{2}^{7}[0,1], W_{2}^{8}[0,1]$ are shown in Table 1. By increasing $m$, the behavior improves. This is an indication of stability on the reproducing Kernel. It is obviously our presented method is more accurate than the Euler-Maclaurin summation formula method [1].
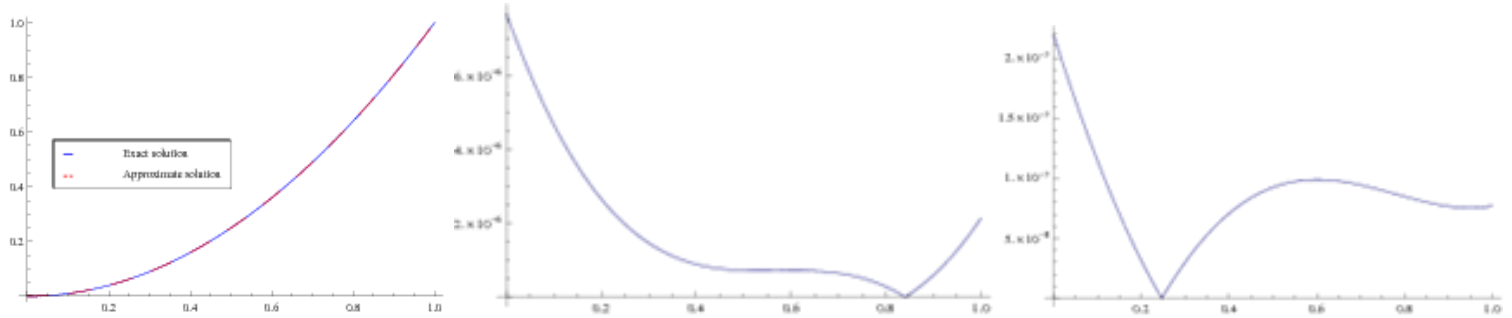

Figure 1. The Figures of the Approximate Solution, the Absolute Errors in $W_{2}^{7}$ and $W_{2}^{8}$, Respectively Left to Right

Table 1. Numerical Results of Example 4.1.

\begin{tabular}{ccc}
\hline Node & $\left|u_{10}(x)-u(x)\right|_{W_{2}^{7}}$ & $\left|u_{10}(x)-u(x)\right|_{W_{2}^{8}}$ \\
\hline 0.0 & $7.67855 \mathrm{E}-6$ & $2.19411 \mathrm{E}-7$ \\
0.1 & $4.66849 \mathrm{E}-6$ & $1.14427 \mathrm{E}-7$ \\
0.2 & $2.65571 \mathrm{E}-6$ & $3.12565 \mathrm{E}-8$ \\
0.3 & $1.46256 \mathrm{E}-6$ & $3.01542 \mathrm{E}-8$ \\
0.4 & $8.89451 \mathrm{E}-7$ & $7.06685 \mathrm{E}-8$ \\
0.5 & $7.17540 \mathrm{E}-7$ & $9.23934 \mathrm{E}-8$ \\
0.6 & $7.14806 \mathrm{E}-7$ & $9.88244 \mathrm{E}-8$ \\
0.7 & $6.45315 \mathrm{E}-7$ & $9.43676 \mathrm{E}-8$ \\
0.8 & $2.81128 \mathrm{E}-7$ & $8.48212 \mathrm{E}-8$ \\
0.9 & $5.85169 \mathrm{E}-7$ & $7.66356 \mathrm{E}-8$ \\
1.0 & $2.12987 \mathrm{E}-7$ & $7.69541 \mathrm{E}-8$ \\
\hline
\end{tabular}

Example 4.2. We consider the following weakly singular Fredholm integral Equation [1]:

$$
u(x)=e^{x}-4.0602+\int_{0}^{1} \frac{u(t)}{\sqrt{1-t}} \mathrm{~d} t, \quad 0 \leq x \leq 1,
$$

the corresponding exact solution is given by $u(x)=e^{x}$.

Let $n=7$ and applying the reproducing kernel method. The comparison between the exact solution and the approximate solution and the absolute errors in spaces $W_{2}^{8}[0,1], W_{2}^{9}[0,1]$ are graphically shown in 
Figure 2, respectively. The absolute errors between $u(x)$ and $u_{10}(x)$ in spaces $W_{2}^{8}[0,1], W_{2}^{9}[0,1]$ are shown in Table 2. By increasing $m$, the behavior improves. This is an indication of stability on the reproducing Kernel. It is obviously our presented method is more accurate than the Euler-Maclaurin summation formula method [1].
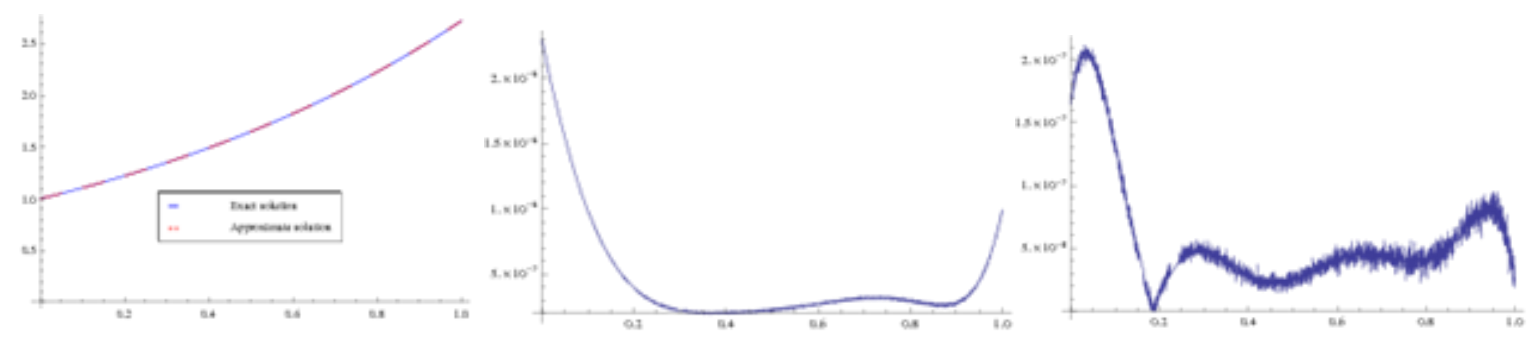

Figure 2. The Figures of the Approximate Solution, the Absolute Errors in $W_{2}^{8}$ and $W_{2}^{9}$, Respectively Left to Right

Table 2. Numerical Results of Example 4.2.

\begin{tabular}{ccc}
\hline Node & $\left|u_{10}(x)-u(x)\right|_{W_{2}^{8}}$ & $\left|u_{N}(x)-u(x)\right|_{W_{2}^{9}}$ \\
\hline 0.0 & $2.29568 \mathrm{E}-6$ & $1.66515 \mathrm{E}-7$ \\
0.1 & $9.78099 \mathrm{E}-7$ & $1.30769 \mathrm{E}-7$ \\
0.2 & $3.95911 \mathrm{E}-7$ & $1.49509 \mathrm{E}-8$ \\
0.3 & $2.23955 \mathrm{E}-7$ & $5.36106 \mathrm{E}-8$ \\
0.4 & $2.01035 \mathrm{E}-7$ & $2.94664 \mathrm{E}-8$ \\
0.5 & $2.27987 \mathrm{E}-7$ & $2.15008 \mathrm{E}-8$ \\
0.6 & $2.71515 \mathrm{E}-7$ & $4.34800 \mathrm{E}-8$ \\
0.7 & $3.03137 \mathrm{E}-7$ & $4.84487 \mathrm{E}-8$ \\
0.8 & $2.97588 \mathrm{E}-7$ & $3.17069 \mathrm{E}-8$ \\
0.9 & $2.76424 \mathrm{E}-7$ & $7.47239 \mathrm{E}-8$ \\
1.0 & $9.86883 \mathrm{E}-7$ & $2.21194 \mathrm{E}-8$ \\
\hline
\end{tabular}

\section{CONCLUSION}

In this paper, we established a method to find numerical solutions of the Fredholm integral equations with a weakly singular kernel. We used the Taylor series to remove singularity and solved some examples with our proposed method. According to the examples solved in two different spaces, by increasing $m$, the behavior improves. This is an indication of stability on the reproducing Kernel. The results from the numerical examples show that the present method is accurate and reliable for solving these equations.

\section{REFERENCES}

[1] R. Behzadi, E. Tohidi, F. Toutounian, "Numerical solution of weakly singular Fredholm integral equations via generalization of the Euler-Maclaurin summation formula," Journal of Taibah University for Science, vol. 8, pp. 199-205, 2014.

[2] J. Biazar, M. Eslami, H. Aminikhah, "Application of homotopy perturbation method for systems of Volterra integral equations of the first kind," Chaos, Solitons \& Fractals, vol. 42, pp. 3020-3026, 2009.

[3] J. Biazar, M. Eslami, M.R. Islam, "Differential transform method for special systems of integral equations," Journal of King Saud University-Science, vol. 24, pp. 211-214, 2012.

[4] A. Pedas, E. Tamme, "Discrete Galerkin method for Fredholm integro-differential equations with weakly singular kernels," Journal of Computational and Applied Mathematics, vol. 213, pp. 111-126, 2008.

[5] J. Biazar, M. Eslami, "Modified HPM for solving systems of Volterra integral equations of the second kind," Journal of King Saud University-Science, vol. 23, pp. 35-39, 2011.

[6] L. Zhu, Y. Wang, "Numerical solutions of Volterra integral equation with weakly singular kernel using SCW method," Applied Mathematics and Computation, vol. 260, pp. 63-70, 2015.

[7] Y. Chen, T. Tang, "Spectral methods for weakly singular Volterra integral equations with smooth solutions," Journal of Computational and Applied Mathematics, vol. 233, pp. 938-950, 2009.

[8] C. Lubich, "Fractional linear multi-step methods for Abel-Volterra integral equations of the second kind," Mathematics of Computation, vol. 45, pp. 463-469, 1985. 
[9] Z. Xiao-yong, "Jacobi spectral method for the second-kind Volterra integral equations with a weakly singular kernel," Applied Mathematical Modelling vol. 39, pp. 4421-4431, 2015.

[10] Y. Chen, T. Tang, "Convergence analysis of the Jacobi spectral-collocation methods for Volterra integral equations with a weakly singular kernel," Mathematics of Computation, vol. 79, pp.147-167, 2010.

[11] E. Babolian, A.A. Hajikandi, "The approximate solution of a class of Fredholm integral equations with a weakly singular kernel," Journal of Computational and Applied Mathematics, pp. 235, pp. 1148-1159, 2011.

[12] M. Federson, R. Bianconi, L. Barbanti, "Linear Volterra integral equations as the limit of discrete systems," Acta Mathematicae Applicatae Sinica, vol. 20, pp. 623-640, 2004.

[13] T. Tang, X. Xu, J. Cheng, "On spectral methods for Volterra type integral equations and the convergence analysis," Journal of Computational Mathematics, vol. 26, pp. 825-837, 2008.

[14] S. Kumar, A. Kumar, D. Kumar, J. Singh, A. Singh, "Analytical solution of Abel integral equation arising in astrophysics via Laplace transform," Journal of the Egyptian Mathematical Society, vol. 23, pp. 102-107, 2015.

[15] Z. Chen, Y.F. Zhou, "An efficient algorithm for solving Hilbert type singular integral equations of the second kind," Computers \& Mathematics with Applications, vol. 58, pp. 632-640, 2009.

[16] H. Du, M. Cui, "Approximate solution of the Fredholm integral equation of the first kind in a reproducing kernel Hilbert space," Applied Mathematics Letters, vol. 21, pp. 617-623, 2008.

[17] H. Du, J. Shen, "Reproducing kernel method of solving singular integral equation with cosecant kernel," Journal of Mathematical Analysis and Applications, vol. 348, pp. 308-314, 2008.

[18] M-Q. Xu, Y-Z. Lin, "Simplified reproducing kernel method for fractional differential equations with daley," Applied Mathematics Letters, vol 52, pp. 156-161, 2016.

[19] S. Abbasbandya, B. Azarnavid, M.S. Alhuthali, "A shooting reproducing kernel Hilbert space method for multiple solutions of nonlinear boundary value problems," Journal of Computational and Applied Mathematics, vol. 279, pp. 293-305, 2015.

[20] M. Ghasemi-Kuzehkanan, M. Fardi, R.K. Ghaziani, "Numerical solution of nonlinear delay differential equations of fractional order in reproducing kernel Hilbert space," Applied Mathematics and Computation, vol. 268, pp. 815-831, 2015.

[21] W. Jiang, T. Tian, "Numerical solution of nonlinear Volterra integro-differential equations of fractional order by the reproducing kernel method," Applied Mathematical Modelling, vol. 39, pp. 4871-4876, 2015.

[22] F.Z. Geng, S.P. Qian, "Modified reproducing kernel method for singularly perturbed boundary value problems with a delay," Applied Mathematical Modelling, vol. 39, pp. 5592-5597, 2015.

[23] H. Du, G. Zhao, C. Zhao, "Reproducing kernel method for solving Fredhom integro-differential equations with weakly singularity," Journal of Computational and Applied Mathematics, vol. 255, pp. 122-132, 2014.

[24] F.Z. Geng, S.P. Qian, S. Li, "A numerical method for singularly perturbed turning point problems with an interior layer," Journal of Computational and Applied Mathematics, vol. 255, pp. 97-105, 2014.

[25] T. Jordão,V.A. Menegatto, "Weighted Fourier-Laplace transforms in reproducing kernel Hilbert spaces on the sphere," Journal of Mathematical Analysis and Applications, vol. 411, pp. 732-741, 2014. 